\title{
Cosmic biology in perspective
}

\author{
N.C. Wickramasinghe ${ }^{1,2}$. Dayal T. Wickramasinghe ${ }^{3}$. Christopher A. Tout ${ }^{4,5} \cdot$ John C. Lattanzio $^{5}$ - Edward J. Steele ${ }^{6,7}$
}

Received: 6 September 2019 / Accepted: 13 November 2019 / Published online: 20 November 2019

(c) The Author(s) 2019

\begin{abstract}
A series of astronomical observations obtained over the period 1986 to 2018 supports the idea that life is a cosmic rather than a purely terrestrial or planetary phenomenon. These include (1) the detection of biologically relevant molecules in interstellar clouds and in comets, (2) mid-infrared spectra of interstellar grains and the dust from comets, (3) a diverse set of data from comets including the Rosetta mission showing consistency with biology and (4) the frequency of Earth-like or habitable planets in the Galaxy. We argue that the conjunction of all the available data suggests the operation of cometary biology and interstellar panspermia rather than the much weaker hypothesis of comets being only the source of the chemical building blocks of life. We conclude with specific predictions on the properties expected of extra-terrestrial life if it is discovered on Enceladus, Europa or beyond. A radically different bio-
\end{abstract}

$\triangle$ C.A. Tout

cat@ast.cam.ac.uk

1 Buckingham Centre for Astrobiology, University of Buckingham, Verney Park Campus, London Road, Buckingham MK18 1AD, UK

2 Centre for Astrobiology, University of Ruhuna, Matara, Sri Lanka

3 Mathematical Sciences Institute, The Australian National University, Canberra, ACT 0200, Australia

4 Institute of Astronomy, The Observatories, Madingley Road, Cambridge CB3 OHA, UK

5 Monash Centre for Astrophysics, School of Physics and Astronomy, Monash University, 10 College Walk, Clayton, VIC 3168, Australia

6 CY O’Connor Village Foundation, 4D, 11A Erade Drive, Piara Waters, WA 6112, Australia

7 Melville Analytics Pty Ltd, Level 7, 14 Queens Road, Melbourne, VIC 3004, Australia chemistry elsewhere can be considered as a falsification of the theory of interstellar panspermia.

Keywords Comets-interstellar · Molecules-interstellar . Grains-panspermia

\section{Introduction}

The essence of biological evolution confined to the Earth is that the accumulation of random copying errors in a genome leads, after the lapse of very many generations, to the emergence of new phenotypes, new species, new orders, new classes. It is postulated that, in the process of replication, a random mutation, that is favoured for survival, occurs and is locked into the genome. Subsequently another random mutation is similarly selected for survival and locked into the germ-line, and the process repeats with no external input required. However, the failure thus far to synthesise living cells nor indeed any approach to a living system de novo in the laboratory after over five decades of serious effort is discouraging (Deamer 2012).

The sufficiency of this neo-Darwinian scheme operating in isolation may be called into question by the observation that, following the first appearance of bacterial life (prokaryotes) on the Earth between 4.1 and 4.2 Gyr ago (Bell et al. 2015), there is a protracted geological period of stasis lasting for about $2 \mathrm{Gyr}$ during which no further evolution is evident. During this period, bacteria and archaea remain the sole life forms on the Earth. The oldest evidence, from molecular data, of further evolution to eukaryotes is found only 2 Gyr ago (Brocks 1999; Strother et al. 2011). Eukaryotes differ fundamentally from bacteria and archaea. Besides being larger in size, eukaryotes possess nuclei and organelles, 
which are thought to have been symbiotic bacteria, and they can reproduce sexually.

Following the appearance of eukaryotes on the Earth at $2 \mathrm{Gyr}$ there is another protracted period in which they remain essentially unaltered. This is also difficult to explain if conventional Earth-centred evolution is the main driving force. Some attempts at multicellular cooperation appear in the form of sheets or filaments but these experiments appear to have come to a dead end (Knoll 2011). The next major innovation in terrestrial biology is the emergence of multicellular life, small animals, and this comes only after another long period of stasis lasting for some 1.5 Gyr until the Cambrian explosion about $540 \mathrm{Myr}$ ago. Again we can note that 1.5 Gyr of neo-Darwinian evolution showed no signs of any progress towards multi-cellularity. Subsequent progress in biological evolution also seems to be punctuated by a series of sharp spikes of speciation as well as of extinctions extending arguably to recent geological epochs (Steele et al. 2018). The currently favoured explanation for this is that mass extinction opens up many opportunities for new life.

It has been estimated that the solar system has experienced dynamical encounters with 5 to 10 giant molecular clouds throughout the course of its history, passing within $5 \mathrm{pc}$ of star-forming nebulae on the average about once every 100 Myr. Numerical simulations with standard $N$-body techniques have led to the conclusion that the ingress of comets into the inner solar system is increased by factors of about 100 during each such encounter (Wickramasinghe et al. 2009). Such enhanced collision rates with comets have the potential to introduce non-solar system biological genetic material. The neo-Darwinian scheme, augmented by horizontal gene transfer during such events, may lead to a general accord with patterns of biological evolution that are witnessed on the Earth (Wallis and Wickramasinghe 2004). The same process can be extended to take place on a Galactic scale between the 10 thousand million or so habitable planetary systems that are now thought to exist in our Galaxy alone (Kopparapu 2013).

Within the solar system itself, and on the Earth in particular, the evolution of life would be controlled by a combination of processes, random encounters with dense star/planet forming clouds in the course of its motion through the Galaxy and the more or less periodic incursions of biologically laden material from comets within the solar system itself. In this way it may be possible to account for both random events, involving long periods of stasis, such as we have discussed, as well as periodic events that punctuate the evolution of life.

\section{Interstellar carbon}

It is now accepted that interstellar dust includes a component of organic grains (Wickramasinghe 1974). In partic- ular, organic grains constitute an integral part of progenitor clouds, similar to the presolar nebula, from which the Sun and planets formed $4.8 \mathrm{Gyr}$ ago. Icy cometesimals are the first condensed bodies that formed in the outer regions of such a nebula and these bodies can be assumed to have mopped up a large fraction of interstellar organics. Such organic molecules would then have been available for delivery via collisions of comets to the inner planets of the solar system including Earth.

\subsection{Organic molecules in space}

Beyond the attribution of hydrocarbon structures in the infrared spectra of Galactic sources to interstellar dust (Sect. 3.1), the definite detections of specific organic molecular species by millimetre wave and radio observations have revealed well over 150 such molecules (Kwok 2016; Thaddeus 2006). They include a branched carbon back-bone isopropyl cyanide, the amino acid glycine, vinyl alcohol, ethyl formate, naphthalene and the chiral molecule propylene oxide (Kuan 2003).

From the 1930s onwards observed spectra of reddened stars have shown a large number of unidentified diffuse interstellar bands (DIBs) extending over visual and near IR wavelengths (Herbig 1995). Complex organic molecules, including even molecules related to chlorophyll, have been proposed but conclusive identification of a specific carrier has remained elusive (Johnson 2006). Two spectral lines at $9577 \AA$ and $9632 \AA$ match a singly charged fullerine $\mathrm{C}_{60}^{+}$ (Campbell et al. 2015), although such an identification of only 2 out of some 400 similar lines can be questioned. More recently there have been claims for the presence of other features attributed to fulleranes in the spectrum of the protoPNe IRAS01005+7910 (Zhang et al. 2016). Fullerine cages that have also been discovered in carbonaceous chondrites provide an additional line of evidence.

The organic molecules so far discovered in interstellar space have prompted astrobiologists to consider seriously the proposition that they may somehow be connected with the origin of terrestrial life. However, even if the appropriate organic molecules are supplied to Earth's oceans where they can contribute to a canonical primordial soup, there remains the improbability of small-scale abiogenesis (Hoyle and Wickramasinghe 1981). For this reason, Crick and Orgel (1973) advocated a directed panspermia where life first originated somewhere else in the larger Universe.

\subsection{The origin of interstellar grains}

The question of the nature and mode of origin of interstellar grains has been addressed since the 1930s (Lynds and Wickramasinghe 1968; Draine 2003). The work of Oort 
and van de Hulst (1946) laid the foundations of modern research on grain formation and grain mantle growth in interstellar clouds. However, difficulties associated with the nucleation of dust in low-density interstellar clouds led to a serious discussion of alternative sites of grain formation. Hoyle and Wickramasinghe $(1962,1969)$ argued that mass outflows from cool giant stars, in particular carbon stars, provide ideal conditions for the nucleation and growth of refractory grains. Shortly afterwards supernovae were proposed as a comparable source of refractory dust (Hoyle and Wickramasinghe 1970).

Both direct observations and theory have now converged to leave little doubt that mass outflows from asymptotic giant branch (AGB) stars, including carbon stars, as well as supernovae, are sources of refractory dust in interstellar space. However, by analysing the quantity of dust in the Galaxy as well as in external galaxies (SMC, LMC, Andromeda satellites), it is possible to set stringent limits on the contributions from different stellar dust sources (Draine 2009). Matsuura et al. (2009) investigated the global mass budget in the LMC and identified a missing dust-mass problem. The accumulated dust mass from AGB stars and $\mathrm{SNe}$ was estimated to be significantly lower than observed in the ISM. A similar conclusion was reached by Zhu et al. (2015) who also included common envelope ejecta and by Srinivasan et al. (2016) from studies of the SMC. De Looze et al. (2016) investigated the origin of interstellar dust in the Andromeda dwarf galaxies and concluded that the observed dust content is an order of magnitude higher than expected from AGB stars and SNe. It now appears that less than 10 per cent of the dust in all cases can be attributed to AGB stars and supernovae, so requiring the bulk of interstellar dust to form by grain mantle growth in the denser clouds. With a wide range of local conditions, cloud densities in particular, a uniform grain composition as well as size is then difficult to explain. It is also not clear how grains with large organic or inorganic mantles can be effectively dispersed into the diffuse ISM where the extinction of light from distant stars $(d>1 \mathrm{kpc})$ is observed. In Sect. 3 we shall argue that biologically generated particles could account for the bulk of the interstellar grains which possess a more or less invariant composition and size.

The contribution to interstellar grains from the biological model (discussed in Sect. 2.5), namely biological dust, can be estimated by noting that the present Oort cloud in our solar system, comprised of $10^{12}$ comets, is generally assumed to have a loss rate to interstellar space of about $10^{11}$ comets every $10^{9} \mathrm{yr}$ (Weissman 1983). This is equivalent to the dispersal of bacterial dust from about 100 comets every year. Assuming a 10 per cent mass fraction of bacterial cells in comets of typical radius of about $10 \mathrm{~km}$, the rate of injection of bacterial grains from our solar system into the interstellar medium is $10^{19} \mathrm{~g} \mathrm{yr}^{-1}$. With a total Galactic population of

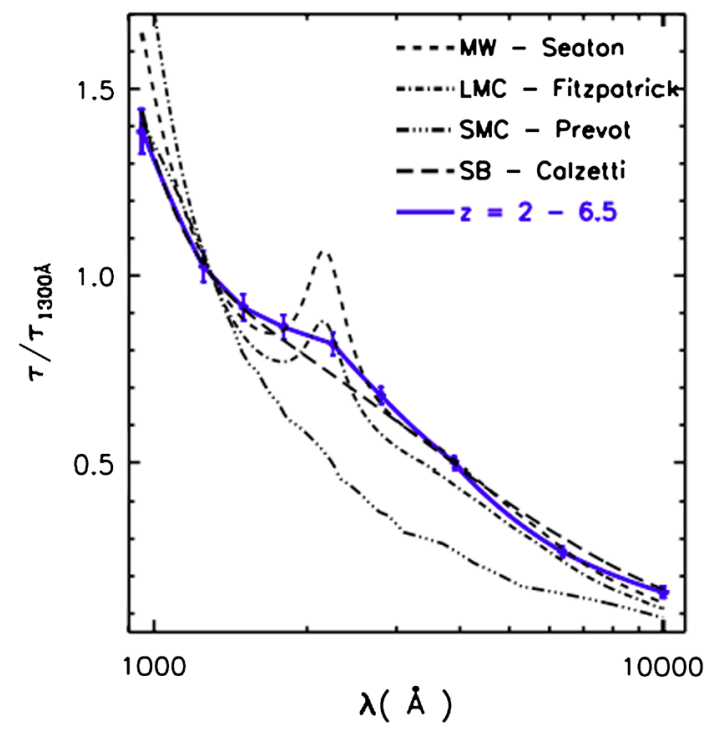

Fig. 1 Extinction curves for galaxies of various redshift. Milky Way (MW) (Seaton 1979), LMC (Fitzpatrick 1986), SMC (Prevot et al. 1984), local starburst galaxies (Calzetti et al. 2000). The compilation is due Scoville et al. (2015)

$10^{11}$ (G dwarf) solar-like stars each endowed with a similar Oort cloud of comets, the total rate of replenishment of bacterial dust into the ISM is $10^{31} \mathrm{~g} \mathrm{yr}^{-1}$. Distributed throughout the volume of the ISM, about $10^{66} \mathrm{~cm}^{3}$, this gives a rate of increase of grain density of $10^{-35} \mathrm{~g} \mathrm{~cm}^{-3} \mathrm{yr}^{-1}$. Over a typical turn-over time of the interstellar medium, $3 \times 10^{9} \mathrm{yr}$, this leads to a mean density of $3 \times 10^{-26} \mathrm{~g} \mathrm{~cm}^{-3}$, which is essentially most of the interstellar dust. Notwithstanding the approximate nature of these estimates, it would appear that the postulated bacterial grains could make a significant, perhaps even dominant, contribution to solid particles in the diffuse interstellar medium.

\subsection{Constraints from interstellar extinction}

The visual extinction of starlight in the solar vicinity, predominantly arising from scattering rather than absorption, amounts to about $1.8 \mathrm{mag} \mathrm{kpc}^{-1}$ (Lynds and Wickramasinghe 1968). This demands a total mass density of grains in the form of silicates, ices and carbonaceous/organic grains with optimum radii to produce visual extinction (mainly owing to scattering) of approximately $0.3 \mu \mathrm{m}$. This material accounts for over 30 per cent of the available $\mathrm{C}, \mathrm{O}$ and $\mathrm{Si}$ in the interstellar medium (Hoyle and Wickramasinghe 1991). Grains growing much larger mantles in the dense interstellar clouds are excluded by this criterion.

The wavelength dependence of extinction has continued to provide stringent constraints on the dust and, for any particular model, such as graphite-silicate mixtures, the particle size spectrum and optical properties need to be finely tuned to agree with the more or less invariant shape of the inter- 
stellar extinction curve (Draine 2003). This interstellar extinction curve (Fig. 1) has been extended both with respect to the wavelengths covered and the distances of sources examined. For wavelengths longward of $3000 \AA$ it is known to be more or less invariant compared to that for shorter ultraviolet wavelengths (Hoyle and Wickramasinghe 1991). The main variation in the ultraviolet is in the slope and the extent of a broad symmetric hump in extinction always centred at $2175 \AA$ Ax Extinction curves for the LMC and the SMC have been observed since 1980 (Gordon et al. 2003). That for the LMC is found to be more or less identical to the Galactic extinction curve while that for the SMC is different to the extent that it has only a weak or non-existent $2175 \AA$ bump. Extinction curves have also recently been extended to very distant galaxies (Scoville et al. 2015; Noll et al. 2009).

It has been widely assumed that the most plausible explanation for the $2175 \AA$ feature of interstellar dust involves a graphite particle model (Hoyle and Wickramasinghe 1962). Wickramasinghe and Guillaume (1965) first used laboratory optical constants of graphite, together with the Mie formulae (van de Hulst 1957), to compute the extinction cross-sections of graphite spheres of various radii. All subsequent calculations for a graphite grain model have followed basically the same procedure. An unsatisfactory feature of the graphite particle explanation of the $2175 \AA$ absorption is the requirement that the graphite is in the form of spheres with a finely tuned radius of $0.02 \mu \mathrm{m}$ and, moreover, that it has isotropic optical properties. The latter assumption is not physically realistic because graphite has strongly anisotropic electrical properties. The particular choice of size in all current modelling of this feature is difficult to defend (Hoyle and Wickramasinghe 1991) and also appears inconsistent with recent theoretical predictions of grain sizes from stellar outflows of AGB stars of solar metallicity (Dell'Agli et al. 2017).

A suitable model for the $2175 \AA$ feature must explain (1) the observed invariance of the rest central wavelength independently of red shift (at least up to $z \approx 6$ ) and (2) the evidence that the strength of the feature depends on galactic environment, with more active galaxies showing weaker features (Fig. 1). These requirements are fulfilled by a dispersed macromolecular organic absorber of universal prevalence. In more active galaxies it would be reasonable to assume that the stability of such molecules is compromised to varying degrees. Hence variations of the strength of the $2175 \AA$ feature would be explained.

Polyaromatic hydrocarbons (PAHs) have been identified as possible candidates to explain the invariant wavelength of the $2175 \AA$ ultraviolet absorption feature. Owing to variability of the central wavelength of the band for different PAHs, for such an explanation to be viable, a highly specific distribution of PAHs with varying states of ionization is demanded (Hoyle and Wickramasinghe 2000; Li and Draine 2001).
We propose that the universality of this feature can be plausibly explained if the macromolecular absorbers are of biological origin. Aromatic and hetero-aromatic groups, $\mathrm{C}_{6}$ rings and rings with $\mathrm{O}$ or $\mathrm{N}$ inclusions, form a large class of organic molecules derived from biology and may represent the most stable break-down products of living systems. Such molecules, including quinolone and quinozoline, are found to have a broad absorption feature resembling the $2175 \AA$ interstellar absorption feature (Hoyle and Wickramasinghe 1977, 1979). It would appear that this feature is best matched by the subset of aromatic functional groups found in biology.

\subsection{Abiotic explanations of IR emission features}

The unidentified infrared bands (UIB's) at 3.3, 6.2, 7.7, 8.6 and $11.3 \mu \mathrm{m}$ seen in the diffuse ISM arising from $\mathrm{CH}$ and $\mathrm{CC}$ vibrational modes of aromatic compounds are usually attributed to PAHs. However this association is by no means universally accepted. When UIBs are seen in astronomical sources they are usually also associated with aliphatic bands at 3.4, 6.9 and $7.3 \mu \mathrm{m}$, so the basic PAH model has been modified to include methyl side groups. As an offshoot of such models, Zhang and Kwok (2015) have proposed a class of mixed organic aromatic/aliphatic nanoparticles (MOANs) as a possible carrier of the UIB bands. Importantly, they also demonstrate that a general agreement between astronomical spectra and spectra of PAH mixtures, as is often found, is not proof of the PAH hypothesis.

As we pointed out earlier, a case can be made for the presence of fullerenes in the ISM but again the question arises as to how they are formed. Their discovery in a proto-planetary nucleus suggests formation on a time-scale of $10^{3} \mathrm{yr}$ which presumably excludes a bottom-up process because of the time required to build macro-molecules from smaller carbon units (Berné et al. 2015). The favoured mechanisms are photon or shock induced processes starting from large hydrocarbon molecules leading to de-hydrogenisation and ejection of $\mathrm{C}_{2}$ (Micelotta et al. 2012; Omont 2016). Possible precursors are PAHs, HAC (hydrogenated amorphous carbon) and MOANs. Biology also produces possible pathways for the generation of fullerenes.

\subsection{Organic molecules in proto-planetary discs}

We expect some fraction of the complex organic solids seen in the diffuse ISM to have originated in proto-planetary nebulae. In addition to the ices that form in situ, dust particles in outflows from stars are entrapped in the collapsing gas clouds, that form proto-planetary discs, then reprocessed in various ways and ejected back into the diffuse ISM. In our alternative biological hypothesis, cometary bodies, moons and planets that also form in these discs are the sites where an incipient biology is amplified and then dispersed through a galaxy. 
Since the work of Sagan and Khare (1979) it has been known that, under suitable conditions, intractable organic polymers are produced on grain surfaces in laboratory experiments devised to simulate conditions in proto-planetary discs (see Muñoz Caro et al. 2002, for more recent work in this area). So the discovery that a large fraction of interstellar carbon in the diffuse interstellar medium is tied up in the form of PAHs and other organic molecules relevant to life has been taken as evidence of such grain surface chemistry or ion-molecule reactions occurring on the surfaces of grains or within interstellar grains irradiated by low-energy electrons and X-rays (Ehrenfreund and Charnley 2000). These are considered as plausible non-biological sources of the organic component of interstellar dust in the diffuse ISM. While it is true that the laboratory polymers formed in this way possess the generic functional groups that are present in all organic material and that similar processes may indeed occur in different interstellar environments, a precise match to astronomical observations must require arbitrary fixing of the relative weights of such functional groups. As we show in Sect. 3.1, the weightings that are required are those that occur naturally through biology. Furthermore, achieving densities of this material exceeding $10^{-7}$ of the ambient hydrogen density in such environments appears to present a serious challenge (Walsh et al. 2014). There are also other difficulties associated with this class of model. The feasibility of production of complex organics by the above processes generally demands a reducing not an oxidizing mixture of molecular ices dominated by $\mathrm{CH}_{4}$. However, since the early work of Oort and van de Hulst (1946), the main component of interstellar ices has been expected to be $\mathrm{H}_{2} \mathrm{O}$ rather than $\mathrm{CH}_{4}$ and the mixture of molecules in the condensed state would possess an overall oxidizing composition. This is indeed consistent with the observation that the dominant form of $\mathrm{C}$ in the gaseous interstellar medium is $\mathrm{CO}$ rather than $\mathrm{CH}_{4}$ or $\mathrm{CN}$. So the conditions under which laboratory experiments have thus far been carried out, involving molecules such as $\mathrm{CH}_{4}$ (for example Esmaili et al. 2017), are inapplicable to the bulk of interstellar ice grains or ice mantles. They could only be justified as being relevant to localised regions of interstellar clouds. Because thermodynamics must always prevail on the large scale, it is unlikely that these processes have a role to play in the bulk chemical transformation of astrophysical ices leading to biologically relevant compounds

\section{Case for biological grains}

A strong argument in favour of biology is that wherever and whenever it operates it is incomparably more efficient than competing abiotic processes. This is apparent when we look at the Earth where 99.999 per cent of all organics have a biological origin (Hoyle and Wickramasinghe 2000). The question is to what extent such processes can operate and convert

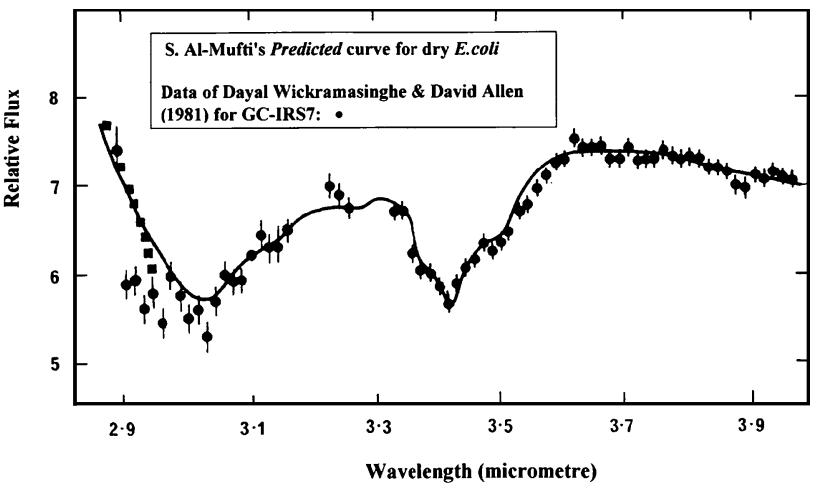

Fig. 2 The first detailed observations of the Galactic Centre infrared source GC-IRS7 (Allen and Wickramasinghe 1981) over the spectral region 2.9 to $3.6 \mu \mathrm{m}$ obtained with the AAT compared with laboratory spectral data for dehydrated bacteria (Hoyle et al. 1982). The $3.4 \mu \mathrm{m}$ feature corresponds to a mixture of aliphatic and aromatic $\mathrm{CH}$ stretching modes and the broad $2.9 \mu \mathrm{m}$ feature to a combination of vibrational/rotational modes of $\mathrm{OH}$ bonds

inorganic matter to organic matter in the physical conditions expected in different astronomical environments. Here, except in the cases of comets and solar system planets and moons, we need to be guided by what we can deduce from remote observations of the properties of interstellar matter.

\subsection{The 2.9 to $4 \mu \mathrm{m}$ absorption spectrum of GC-IRS7}

In contrast to the extinction in the visual band which is mainly due to scattering, the infrared and ultraviolet absorption features of interstellar dust are independent of grain size and yield invaluable information on its molecular composition. The possibility of a largely organic model of interstellar dust became apparent when the first mid-infrared observations of the source GC-IRS7 near the Galactic Centre in the spectral region 2.9 to $4 \mu \mathrm{m}$ with the Anglo-Australian Telescope (AAT) revealed the average properties of dust over a distance scale of some $10 \mathrm{kpc}$ (Allen and Wickramasinghe 1981). The absence of the usually dominant water ice feature at $3.06 \mu \mathrm{m}$ in observations towards most molecular cloud sources demonstrates that the path length towards GC-IRS7 represents the properties of dust in the diffuse ISM. The discrete features stretching from 2.9 to $3.6 \mu \mathrm{m}$ can be identified with a mixture of aliphatic and aromatic $\mathrm{CH}$ stretching modes in an organic solid combined with vibrational/rotational modes of OH bonds. Hoyle et al. (1982) showed that there is a close correspondence between the astronomical data and laboratory data of desiccated bacteria shown in Fig. 2. The total extinction over a $10 \mathrm{kpc}$ path length to the source GC-IRS7 is 0.56 mag. Combining this data with the measured absorption coefficient of desiccated bacteria at $3.4 \mu \mathrm{m}$ of about $750 \mathrm{~cm}^{2} \mathrm{~g}^{-1}$ we find that the smeared out density of absorbing material in interstellar space is $2 \times 10^{-26} \mathrm{~g} \mathrm{~cm}^{-2}$ close to 50 per cent of the density of dust responsible for the visual extinction of starlight. 
The $3.4 \mu \mathrm{m}$ spectral feature in GC-IRS7 stands out in support for the theory of cosmic biology. This feature has now been confirmed by observations of other Galactic Centre sources that do not show a dominant water-ice feature. We note in particular the study of the Quintuplet region by Chiar et al. (2013) who, using the United Kingdom Infrared Telescope (UKIRT), find spectra that are similar to the GCIRS7 spectrum. The small variations can be interpreted as representing varying states of degradation of bacterial grains expected in different environments.

The conclusion is that the bulk of interstellar dust contains a distribution of $\mathrm{CH}$ and $\mathrm{OH}$ bonds, in various configurations, that closely matches biology. The details of such spectroscopic correspondences cannot be claimed to be either unique or incompatible with more conservative dust models (Sect. 3.3). However the existence of such a high fraction of interstellar carbon in the form of organic molecules linked together so as to mimic biology raises a tricky problem relating to the origin of such material. Biology itself is one logical option but, if that is to be rejected, abiotic synthesis is required and the questions of where this occurs and its efficiency have to be answered. The problems associated with one such possibility, namely the synthesis of complex organic molecules in proto-planetary discs, have already been discussed (Sect. 2.5).

\subsection{Extended red emission}

The detection of extended red emission (ERE) over the waveband 6000 to $8000 \AA$ in planetary nebulae (Witt and Schild 1985; Witt et al. 1984) can also be interpreted as evidence for the presence of aromatic molecules which could be of biological origin. This feature has now been ubiquitously observed in a wide variety of dusty objects and regions in our Galaxy and outside. ERE has also been observed in the diffuse interstellar medium (Gordon et al. 1998) and in high latitude Galactic cirrus clouds (Witt et al. 2008). Witt and Vijh (2004) have reviewed abiotic models to interpret ERE emission by a hypothetical photoluminescence process in interstellar dust containing aromatic groups. The absorption of photons at ultraviolet/optical wavelengths is postulated to be followed by electronic transitions associated with the emission of longer-wavelength optical and near-IR photons as a two-stage de-excitation process near the valence band in a semiconductor particle. Although it is widely claimed that ERE is caused by simple, possibly compact, PAHs under various excitation conditions, the actual fits of models to the astronomical data have not been satisfactory (Thaddeus 2006). The fluorescence in fragments of biomaterial such as chloroplasts, cell inclusions including chlorophyll, offers another possibility. Figure 3 compares the fluorescence behaviour of fragmented spinach chloroplasts (Boardman et al. 1966) at
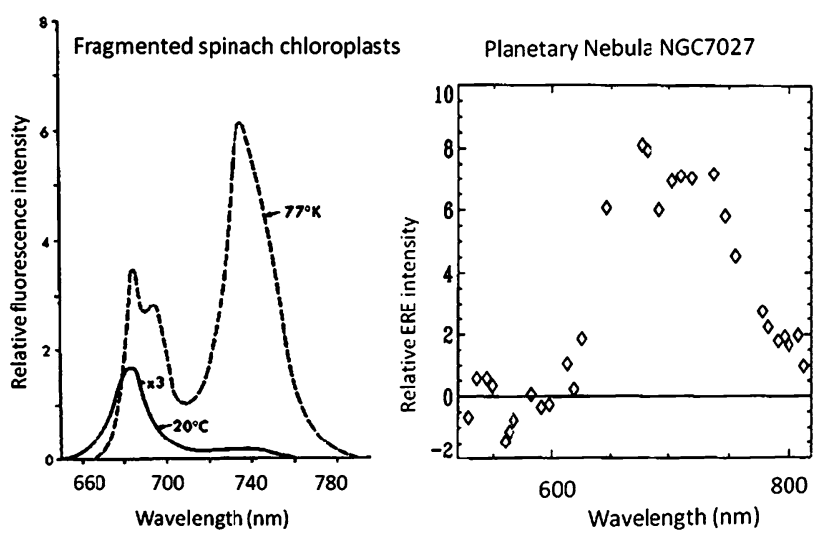

Fig. 3 Left panel: Spectra of fragmented spinach chloroplasts at two temperatures (Boardman et al. 1966). Right panel: A spectrum of ERE excess in NGC7027

different temperatures with the observations of a planetary nebula NGC7027 (Furton and Witt 1992). We note the presence of two temperature dependent broad features centred at about $6800 \AA$ and $7400 \AA$ which may make a contribution to the observed excess in NGC7027. Chloroplasts are not presented here as a definitive or unique identification of the ERE carrier but rather as an illustration of the type of biological PAH that could collectively play a role. It is also worth noting that the biological structures that give rise to ERE could also be responsible for many of the observed UIBs (Smith et al. 2007; Kwok 2009; Kwok and Zhang 2011).

\subsection{Biotic or abiotic origin?}

The question now arises as to the mode of origin of interstellar organic material that appears similar to desiccated biomaterial and its component products. We have already mentioned that the preferred formation mechanisms, that involve grain surface chemistry, grain mantle processing and ion-molecule reactions, although technically feasible, have uncertain efficiencies. The convergence of all such processes to produce a desired end result in terms of satisfactory spectral fits to astronomical data sets is also arbitrary. In one particular instance, the broad $3.4 \mu \mathrm{m}$ absorption profile of the Galactic Centre Quintuplet sources (Sect. 3.1) has been shown to be reproducible with a carefully chosen laboratory mixture of compounds with appropriate aliphatic and aromatic CH stretching modes (Chiar et al. 2013). Whilst the fits to the data cannot be disputed, its applicability to only a limited wavelength region and the ad hoc nature of the weighting factors involved in the synthetic spectrum has no physical basis.

Universal biology on the other hand would set the relative proportions of its main functional chemical units, such as carbohydrates, lipids, nucleic acids and proteins, within narrow limits and their eventual degradation to coal and graphite would also be expected to follow a natural course 
(McFadzean et al. 1989; Okuda et al. 1990; Ishii et al. 1998). Mixtures of biomaterial and inorganic dust from AGB stars and supernovae could in our view offer a plausible explanation of all the available astronomical data.

\section{Comets as a source of interstellar microbiota}

The view that comets and similar icy bodies are unsustainable as microbial habitats is based on the premise that their surface temperatures at heliocentric distances very much greater than $1 \mathrm{AU}$ lead inevitably to hard-frozen conditions. This is not true if radioactive nuclides are taken into account (Hoyle and Wickramasinghe 1978; Wallis 1980). Yabushita (1993) has shown that with the inclusion of radioactive nuclides ${ }^{232} \mathrm{Th},{ }^{238} \mathrm{U}$ and ${ }^{40} \mathrm{~K}$, liquid water domains in comets can be maintained for more than a Gyr after they first condense in the outer regions of the solar nebula. Within such domains, even very small numbers of viable bacterial cells or spores could grow exponentially on a very short timescale. Biological activity occurring in primordial comets at heliocentric distances greater than 20,000 AU can then contribute to ejecta that reach escape velocities from the entire solar system. In our own solar system the total mass of comets and icy planets is about $10^{-2} M_{\odot}$ so we can argue generally that a significant fraction of the $\mathrm{C}, \mathrm{N}$ and $\mathrm{O}$ material that goes into star formation is processed into bacterial type-dust within cometary bodies and ejected back into interstellar space. Within the uncertainties of all the assumptions involved in this argument we do have a process that can produce dust at higher rates than supernovae and AGB stars. Furthermore, in comets approaching perihelion, biological activity could be transiently triggered by solar heating and so lead to the generation of high-pressure pockets of gaseous metabolites that in turn trigger the release of jets of gas and biological particles in their wake (Wickramasinghe et al. 1996). Similar conditions are likely to exist in subsurface lakes in other solar-system planetary bodies including Europa and Enceladus.

\subsection{Biological material in comets}

When Hoyle and Wickramasinghe (1981) first promulgated the idea of cometary panspermia, the supporting evidence was tenuous and appeared far-fetched. There was early evidence from infrared spectroscopy that organic polymeric grains were present in the dust tails of comets and this called into question the Whipple dirty snowball paradigm (Vanýsek and Wickramasinghe 1975). There is also the observation that cometary nuclei can be coal-like with low albedos, in contrast to the expected high albedos of icy surfaces (Cruikshank et al. 1985).

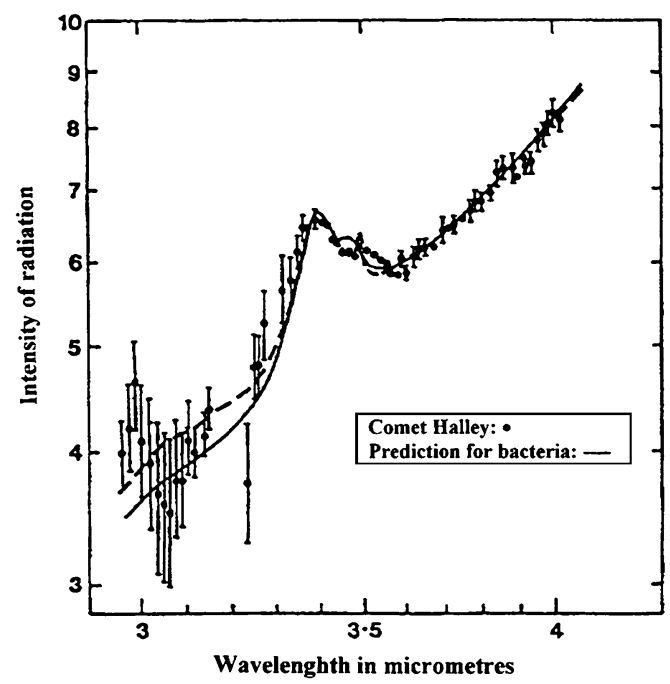

Fig. 4 Emission by the dust coma of Comet Halley observed on March 31, 1986 (points) with the AAT, compared to normalised fluxes for desiccated E-coli at an emission temperature of $320 \mathrm{~K}$. The solid curve is for unirradiated bacteria and the dashed curve is for X-ray irradiated bacteria (Wickramasinghe and Allen 1986; Hoyle and Wickramasinghe 2000)

The idea of cometary microbiology moved swiftly from speculation to quantitative measurement after the last perihelion passage of Comet P/Halley in 1986. The first investigation of a comet in the Space Age (the Giotto mission) marked an important turning point in the history of cometary science. A dark comet surface, darker than the darkest coal, was observed by the Giotto photometry. More importantly, Wickramasinghe and Allen (1986), using the Anglo-Australian Telescope, obtained a 2 to $4 \mu \mathrm{m}$ spectrum of the dust from an outburst of the comet on 31st March 1986. This spectrum showed unequivocal evidence of $\mathrm{CH}$ rotational/vibrational stretching modes indicating complex aromatic and aliphatic hydrocarbon structures, consistent with the expected spectrum of bacterial dust (Hoyle and Wickramasinghe 2000, Fig. 4).

A number of similar discoveries soon followed. The infrared spectrum of Comet Hale-Bopp at 2.9 AU (Crovisier et al. 1997) was shown to match the behaviour of a mixture of microbes with a 10 per cent contribution of silicates predominantly contributing only to the $10 \mu \mathrm{m}$ feature (Wickramasinghe and Hoyle 1999). A very similar spectrum was obtained from the post-impact ejecta in the Deep Impact mission of 2005 (A'Hearn et al. 2005; Lisse et al. 2006). Wickramasinghe et al. (1996) had argued that the prodigious output of organic dust and $\mathrm{CO}$ observed from Comet HaleBopp at $6.5 \mathrm{AU}$, where no cometary activity was normally expected, was indicative of the high-pressure release of material from a liquefied subsurface domain within which microbiology may exist.

Historically the next significant correspondence with biology was to emerge from the Stardust mission which cap- 


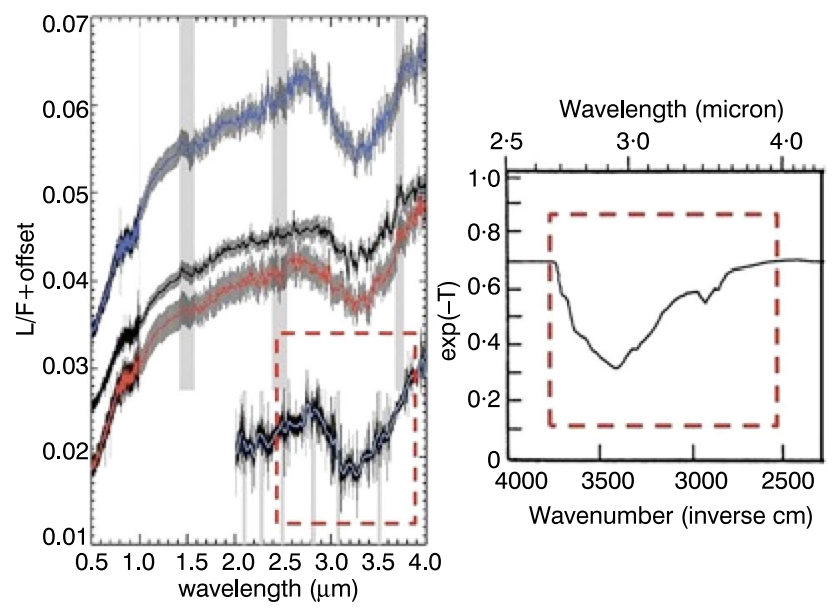

Fig. 5 Left panel: The surface reflectivity spectra of comet $67 \mathrm{P} / \mathrm{C}-\mathrm{G}$ (Capaccioni et al. 2015) taken at different times. Right panel: The transmittance spectrum of desiccated E-coli (cf. Figs. 2 and 4) over approximately the same wavelength range for comparison

tured high speed cometary dust in blocks of aerogel and studied the residues in the laboratory. Amongst these was the most common of amino acids glycine together with a complex mixture of hydrocarbons (Elsila et al. 2009).

The conclusions that can be drawn from these and similar studies, based on space missions to comets, is that the evidence so far does not contradict cometary microbiology. Rather, there are unexpected features that continue to be discovered with the most recent space probes of comets, that are inconsistent with expectations of simple chemistry but are readily explained in terms of cometary biology.

\subsection{Thermochemical equilibrium or biology-new evidence?}

Whenever a distant astronomical body exhibits conditions that can support life the next objective is to look for evidence of molecules or chemistry that may be indicative of life. The model for life that is used is of terrestrial carbonbased life, the only life we know of and available for direct experimentation.

The Rosetta mission's Philae lander has recently provided information about the comet $67 \mathrm{P} / \mathrm{C}-\mathrm{G}$ that appears to be in conflict with the concept of a hard-frozen nonbiological comet. The overall reflectivity spectrum of the surface material of the comet is similar to that of biological material as seen in Fig. 5 (Capaccioni et al. 2015; Wickramasinghe et al. 2015). Jets of water and organics which issue from ruptures and vents in the comet's frozen surface could be due to biological activity occurring within sub-surface liquid pools.

A recent report of $\mathrm{O}_{2}$ along with evidence for the occurrence of water and organics together in the same place
(Bieler et al. 2015) provides further indication of ongoing biological activity. Such a mixture of gases cannot be produced under equilibrium thermodynamic conditions because organics are readily destroyed in an oxidizing environment. The freezing of an initial mixture of compounds, including $\mathrm{O}_{2}$, not in thermochemical equilibrium has been proposed. Although possible, there is no evidence to support such a claim. On the other hand the oxygen/water/organic outflow from the comet can be readily explained on the basis of subsurface microbiology. Photosynthetic microorganisms operating at the low light levels near the surface at perihelion could produce $\mathrm{O}_{2}$ along with organics. The combination of oxygen $\mathrm{O}_{2}$ or $\mathrm{O}_{3}$ (oxidizing) with gases such as methane (reducing) is not permitted in local thermodynamic equilibrium (LTE) so their coincidence in space is possible evidence of biology.

We next turn to the presence of the amino acid glycine and an abundance of phosphorus in the coma of comet 67P/C-G (Altwegg et al. 2016). The very high observed ratio of $\mathrm{P} / \mathrm{C} \approx 10^{-2}$ by number is difficult to reconcile on the basis of volatilization of condensed material of solar composition with $\mathrm{P} / \mathrm{C} \approx 10^{-3}$, particularly when we might expect inorganic phosphorus to be mostly fixed in refractory minerals. On the other hand the $\mathrm{P} / \mathrm{C}$ ratio of organic material found from Rosina can be explained if the material in the coma began as biomaterial, such as virions or bacteria.

Methyl chloride $\left(\mathrm{CH}_{3} \mathrm{Cl}\right)$ and other haloalkanes are a significant source of atmospheric carbon from volatile haloalkanes on Earth. It is mostly produced by oceanic biochemical processes by various macroalgal and other biomasses both in the presence and absence of sunlight (Lobert et al. 1999; Laturnus et al. 1998; Laturnus 2001). Further emission of $\mathrm{CH}_{3} \mathrm{Cl}$ by wood rotting fungi contributes to some of the atmospheric complement of this molecule (Watling and Harper 1998). So the presence of significant amounts of $\mathrm{CH}_{3} \mathrm{Cl}$ and related haloalkanes in space is a possible biomarker, indicating production in habitats similar to those found on Earth. The discovery of this molecule both in interstellar clouds and in comet $67 \mathrm{P} / \mathrm{C}-\mathrm{G}$ (Fayolle et al. 2017) is therefore significant.

Finally we note the intriguing observation that comet Lovejoy emits a prodigious amount of ethanol, equivalent to that in 500 bottles of wine, per second (Biver et al. 2015). This has no explanation in terms of simple chemistry. On the other hand many species of fermenting bacteria can produce ethanol from sugars so this may be another indication that microbial processes operate in comets.

\subsection{Space hardiness of microbes}

The idea that microbial life does not exist in comets still dominates scientific orthodoxy but its rational basis is fast eroding. There is ample evidence that microorganisms 
are space hardy in every conceivable respect (Wickramasinghe 2015; Horneck et al. 2001). There are many types of cryophilic microorganisms that are resistant to freezethaw cycles and it has recently been demonstrated that such microbes exist in arctic ice and permafrost. They can even metabolise at temperatures well below the freezing point of water (Junge et al. 2006).

Early objections to the concept of panspermia were based on the expectation that bacteria and other microorganisms would not survive space travel, high speed entry into a planetary atmosphere or long periods in space (Horneck et al. 2001). However, laboratory experiments have shown that microorganisms can survive hypervelocity impacts (Burchell et al. 2004). Microorganisms with even modest protective mantles against UV radiation, and it seems likely that microorganisms encased in meteorites, can survive in interstellar space for millions of years (Horneck 1993; Horneck et al. 1994, 1995). Microbes have also been shown to survive on the exterior of the surfaces of rockets fired through the atmosphere (Thiel et al. 2014).

The search for incoming bacteria and viruses with balloon-borne equipment lofted to the stratosphere has been carried out for nearly two decades (Harris et al. 2002; Wainwright et al. 2003; Shivaji et al. 2009). Stratospheric air samples recovered from heights ranging from 28 to $41 \mathrm{~km}$ yield evidence of microorganisms but these are widely regarded as most likely to have been lofted from the Earth's surface. Recently Grebennikova et al. (2018) have confirmed the discovery of several microbial species associated with dust on the exterior windows of the International Space Station at a height of $400 \mathrm{~km}$ above the Earth's surface and contamination at source and in the laboratory has been ruled out. The results of PCR (polymerase chain reaction) amplification followed by DNA sequencing and phylogenetic analysis have established the presence bacteria of the genus Mycobacteria and the extremophile genus Delftia associated with deposits of dust. Grebennikova et al. (2018) discuss possible mechanisms for ejecting terrestrial microbes against gravity to such heights in the ionosphere but concede that an origin from outer space remains a possibility.

Far from being the fragile earth-oriented structures they were once considered, microorganisms are incredibly spacehardy. In a review of these and other similar experiments, Burchell (2004) concludes that, with the data at hand, interstellar panspermia can neither be proved nor disproved. It therefore remains a logical option to be evaluated on the basis of other data as we discuss here.

In order for panspermia to operate only a minute fraction of microbes need to survive the transit between one amplification site, such as comets in one planetary system, to another. The exponential replication power of microorganisms is well recognised much to the chagrin of hapless victims of microbial diseases on Earth.

\subsection{Exo-planets and exo-comets}

Recent observations from the Kepler mission have shown that planetary systems are exceedingly common in the Galaxy. The total tally of habitable exoplanets is estimated as upwards of $10^{10}$ (Kopparapu 2013) and, because comets and asteroids form an integral component of our solar system, it is reasonable to presume that such objects are also common in other planetary systems. The detection of exocomets is not easy and only in one case, around the star KIC2542116, is there direct evidence of transiting comets (Rappaport et al. 2018).

A significant fraction, perhaps 30 per cent, of long-period comets that approach perihelion actually leave the solar system on hyperbolic orbits (Hughes 1991). If other exoplanetary systems are similar, and are losing comets at a comparable rate, such comets must be reaching us at a steady rate. The first confirmed interstellar object in our vicinity, Oumuamua (Meech et al. 2017), may be such an object if it is found to be an extremely low albedo comet rather than an asteroid (Wickramasinghe et al. 2018). There are also indications that for the star KIC8462852 the unusual dips in brightness (up to 20 per cent over timescale of days, as well as longer period oscillations that continued to the present day) may be due to obscuration by dust from comets (Wyatt et al. 2018). Further detection of exo-comets will provide necessary evidence for or against the possibility of interstellar panspermia.

\section{Comets and interstellar dust-a re-appraisal}

The data from comets corroborates the hypothesis that similar data relating to the organic composition of interstellar dust, starting with the observations of Wickramasinghe and Allen (1980) and Allen and Wickramasinghe (1981) and subsequent observations of interstellar dust and molecules in different astronomical environments (see Kwok 2016, for a recent review), may have a biological origin. Indeed it has become increasingly apparent in all these cases that it is not possible to exclude biology with remote spectroscopy alone. The spectroscopic signatures of biology or the detritus of biology are, to the first order, very similar to what is seen in astronomy.

It has been claimed that the data currently at hand already contradict the hypothesis of cosmic biology. We now examine these claims one by one.

- One of the key quantities that can be estimated from unmanned space probes of comets is the average composition of outflows. It might be claimed that the near solarsystem type compositions that are found in such studies rule out the possibility of biology in comets (Kwok 2016). 
However, the determination of the average chemical composition of an astronomical environment such as a comet, that must necessarily contain only a small fraction of biological material, cannot provide a discriminant that would either rule in or rule out biology. Other considerations such as we have discussed need to be properly assessed and evaluated.

- A specific objection raised against biology in comets is that no molecules exclusive to biology, such as ATP or DNA, have been discovered. This objection appears to be particularly cogent for the case of comet $67 \mathrm{P} / \mathrm{C}-\mathrm{G}$ where some in situ spectroscopy has been carried out. However, in the absence of any direct life detection experiment on the Philae lander, we have to rely on indirect indicators that are already strong. These include the infrared reflectivity spectra Fig. 5 over the 2 to $4 \mu \mathrm{m}$ region, the phosphorous excess and the non equilibrium $\mathrm{O}_{2}$ abundances. Moreover it should be stressed that biological replication is supposed, according to our model, to occur at some depth below the surface so that surface organics may be mostly comprised of biological metabolites with little in the way of living cells with detectable DNA.

- The match of the interstellar absorption and extinction data to biology is not unique and the same data can be matched with arbitrary mixtures of silicates, graphite and abiotic organic molecules (Draine 2003). However, as we have already noted, in the case of a non-biological explanation, ad hoc assumptions of relative proportions need to be made and the precise invariance of these proportions in different directions in the sky and in various different astronomical and cosmological settings is difficult to justify. As we have discussed, universal biology leads to uniquely defined proportions.

- A prevailing view is that the astronomical explanation is redundant because biologists have a satisfactory and wellproven explanation of abiogenesis on the Earth perhaps in deep sea thermal vents (Sojo et al. 2016). This is not true. All attempts at re-creating abiogenesis in the laboratory have failed. No experimental proof of abiogenesis in a terrestrial setting yet exists (Deamer 2012), so alternatives must be considered.

With the evidence currently at hand, and we confine the discussion to astronomical data alone, the most we can rigorously claim is consistency with a theory of biological grains. However the single unifying concept of cosmic biology remains perhaps the most economical hypothesis to explain the array of facts available to us now. The facts to be combined derive from many diverse disciplines of astronomy, biology, geology and space exploration. An application of Occam's razor prefers such a model. Alternative explanations demand a convolution of physical processes and mechanisms of varying degrees of plausibility.

\section{Some predictions of interstellar panspermia}

Microorganisms on Earth display an incredible range of functional diversity. Particular microbial species colonise particular niches, tolerating extremes of temperature, acidity, salinity, desiccation and high levels of ionizing radiation. Microbial habitats include subterranean ocean caves, hydrothermal vents, polar ice and tropospheric clouds. Throughout this vast range of conditions an amazing biochemical unity prevails pointing unerringly to a single origin. According to interstellar panspermia that event was probably external to our solar system.

If panspermic life is discovered on planetary habitats outside Earth such life is expected to possess the same unity of biochemistry that is found on Earth, the same genetic code, the same DNA and similar biochemistries. Only the habitat selects what is best suited for survival in a similar way to the microorganisms inhabiting the diverse range of habitats on Earth. If independent origin events occur such a correspondence is extremely unlikely. A radically different biochemistry discovered elsewhere would be significant evidence against a universal Galactic panspermia.

In the concordance Big Bang model of the Universe, our observable horizon is estimated to have a radius of about 46 Glyr (Tamara and Lineweaver 2004). Microwave background radiation observations point to the Universe being flat to within 0.4 per cent (Hinshaw et al. 2013). The true spatial extent of the Universe may even be infinite if it is precisely flat. If life originates as a highly improbable event, it is possible that such events are localised within disjoint life domains in the Universe. The cosmological domain in which we happen to find ourselves defines the nature of our idiosyncratic cosmic biology, including its basic biochemistry, as well as its full range of genetic diversity, a range that is only incompletely expressed on Earth. Panspermia reduces the status of the Earth to one of many building sites upon which an already evolved cosmological legacy of life came to be expressed.

History of science has repeatedly shown any theory that requires the Earth to hold a special position in the Universe has proved wrong, the Copernican Principle. The Earth is not the centre of our Universe, nor is our Solar system nor our Milky Way Galaxy. It is therefore extremely unlikely that the Earth can in any way be regarded the centre of life of the Universe. This is particularly so if we take account of recent discoveries of habitable exoplanets with an estimated total of greater than $10^{10}$ in our Galaxy alone.

Our case for panspermia rests on a few simple ideas. First the very early but punctuated origin of life on Earth seems difficult to explain with a slow mutation and selection alone. Secondly biological molecules produced by terrestrial life provide a simple explanation for what is observed in our Solar System, our Galaxy and further reaches of the Universe. 
Such uniformity is only possible if life originated from a single or a number of very similar specialised environments. That it has spread from such a single source provides a simple and easily falsified explanation of a number of otherwise difficult to explain observations.

We conclude by reminding the reader of the prevailing state of science before the Ptolemaic solar system was finally abandoned. Historian of science Thomas Kuhn (1962) wrote,

\section{"The state of Ptolemaic [Earth-centred] astronomy was a scandal before Copernicus' announcement. ... Given a particular discrepancy, astronomers were invariably able to eliminate it by making some partic- ular adjustment in Ptolemy's system of compounded circles. But as time went on, a man looking at the net result of the normal research effort of many as- tronomers could observe that astronomy's complexity was increasing far more rapidly than its accuracy and that a discrepancy corrected in one place was likely to show up in another."}

Acknowledgements CAT thanks Churchill College for his fellowship. Part of this work was completed when CAT was visiting the Mathematical Sciences Institute of the Australian National University and Monash University as a Kevin Westfold distinguished visitor.

Publisher's Note Springer Nature remains neutral with regard to jurisdictional claims in published maps and institutional affiliations.

Open Access This article is distributed under the terms of the Creative Commons Attribution 4.0 International License (http://creative commons.org/licenses/by/4.0/), which permits unrestricted use, distribution, and reproduction in any medium, provided you give appropriate credit to the original author(s) and the source, provide a link to the Creative Commons license, and indicate if changes were made.

\section{References}

A'Hearn, M.F., Belton, M.J.S., Delamere, W.A., Kissel, J., Klaasen, K.P., McFadden, L.A., Meech, K.J., Melosh, H.J., Schultz, P.H., Sunshine, J.M., Thomas, P.C., Veverka, J., Yeomans, D.K., Baca, M.W., Busko, I., Crockett, C.J., Collins, S.M., Desnoyer, M., Eberhardy, C.A., Ernst, C.M., Farnham, T.L., Feaga, L., Groussin, O., Hampton, D., Ipatov, S.I., Li, J.-Y., Lindler, D., Lisse, C.M., Mastrodemos, N., Owen, W.M., Richardson, J.E., Wellnitz, D.D., White, R.L.: Science 310, 258 (2005)

Allen, D.A., Wickramasinghe, D.T.: Nature 294, 239 (1981)

Altwegg, K., Balsiger, H., Bar-Nun, A., Berthelier, J.-J., Bieler, A., Bochsler, P., Briois, C., Calmonte, U., Combi, M.R., Cottin, H., De Keyser, J., Dhooghe, F., Fiethe, B., Fuselier, S.A., Gasc, S., Gombosi, T.I., Hansen, K.C., Haessig, M., Jäckel, A., Kopp, E., Korth, A., Le Roy, L., Mall, U., Marty, B., Mousis, O., Owen, T., Reme, H., Rubin, M., Semon, T., Tzou, C.-Y., Waite, J.H., Wurz, P.: Sci. Adv. 2, e1600285 (2016)

Bell, E.A., Boehnke, P., Harrison, T.M., Mao, W.L.: Proc. Natl. Acad. Sci. 112, 14518 (2015)

Berné, O., Montillaud, J., Joblin, C.: Astron. Astrophys. 577, A133 (2015)
Bieler, A., Altwegg, K., Balsiger, H., Bar-Nun, A., Berthelier, J.-J., Bochsler, P., Briois, C., Calmonte, U., Combi, M., de Keyser, J., van Dishoeck, E.F., Fiethe, B., Fuselier, S.A., Gasc, S., Gombosi, T.I., Hansen, K.C., Hässig, M., Jäckel, A., Kopp, E., Korth, A., Le Roy, L., Mall, U., Maggiolo, R., Marty, B., Mousis, O., Owen, T., Rème, H., Rubin, M., Sémon, T., Tzou, C.-Y., Waite, J.H., Walsh, C., Wurz, P.: Nature 526, 678 (2015)

Biver, N., Bockelée-Morvan, D., Moreno, R., Crovisier, J., Colom, P., Lis, D.C., Sandqvist, A., Boissier, J., Despois, D., Milam, S.N.: Sci. Adv. 1, 1500863 (2015)

Boardman, N.K., Thorne, S.W., Anderson, J.M.: Proc. Natl. Acad. Sci. 56, 586 (1966)

Brocks, J.J.: Science 285, 1033 (1999)

Burchell, M.J.: Int. J. Astrobiol. 3, 73 (2004)

Burchell, M.J., Mann, J.R., Bunch, A.W.: Mon. Not. R. Astron. Soc. 352, 1273 (2004)

Calzetti, D., Armus, L., Bohlin, R.C., Kinney, A.L., Koornneef, J., Storchi-Bergmann, T.: Astrophys. J. 533, 682 (2000)

Campbell, E.K., Holz, M., Gerlich, D., Maier, J.P.: Nature 523, 322 (2015)

Capaccioni, F., Coradini, A., Filacchione, G., Erard, S., Arnold, G., Drossart, P., De Sanctis, M.C., Bockelee-Morvan, D., Capria, M.T., Tosi, F., Leyrat, C., Schmitt, B., Quirico, E., Cerroni, P., Mennella, V., Raponi, A., Ciarniello, M., McCord, T., Moroz, L., Palomba, E., Ammannito, E., Barucci, M.A., Bellucci, G., Benkhoff, J., Bibring, J.P., Blanco, A., Blecka, M., Carlson, R., Carsenty, U., Colangeli, L., Combes, M., Combi, M., Crovisier, J., Encrenaz, T., Federico, C., Fink, U., Fonti, S., Ip, W.H., Irwin, P., Jaumann, R., Kuehrt, E., Langevin, Y., Magni, G., Mottola, S., Orofino, V., Palumbo, P., Piccioni, G., Schade, U., Taylor, F., Tiphene, D., Tozzi, G.P., Beck, P., Biver, N., Bonal, L., Combe, J.-P., Despan, D., Flamini, E., Fornasier, S., Frigeri, A., Grassi, D., Gudipati, M., Longobardo, A., Markus, K., Merlin, F., Orosei, R., Rinaldi, G., Stephan, K., Cartacci, M., Cicchetti, A., Giuppi, S., Hello, Y., Henry, F., Jacquinod, S., Noschese, R., Peter, G., Politi, R., Reess, J.M., Semery, A.: Science 347, aaa0628 (2015)

Chiar, J.E., Tielens, A.G.G.M., Adamson, A.J., Ricca, A.: Astrophys. J. 770, 78 (2013)

Crick, F.H.C., Orgel, L.E.: Icarus 19, 341 (1973)

Crovisier, J., Leech, K., Bockelee-Morvan, D., Brooke, T.Y., Hanner, M.S., Altieri, B., Keller, H.U., Lellouch, E.: Science 275, 1904 (1997)

Cruikshank, D.P., Hartmann, W.K., Tholen, J.T.: Nature 315, 122 (1985)

De Looze, I., Baes, M., Bendo, G.J., Fritz, J., Boquien, M., Cormier, D., Gentile, G., Kennicutt, R.C., Madden, S.C., Smith, M.W.L., Young, L.: Mon. Not. R. Astron. Soc. 459, 3900 (2016)

Deamer, D.: First Life. University of California Press, Oakland (2012)

Dell'Agli, F., García-Hernández, D.A., Schneider, R., Ventura, P., La Franca, F., Valiante, R., Marini, E., Di Criscienzo, M.: Mon. Not. R. Astron. Soc. 467, 4431 (2017)

Draine, B.T.: Annu. Rev. Astron. Astrophys. 41, 241 (2003)

Draine, B.T.: In: Henning, T., Grün, E., Steinacker, J. (eds.) Cosmic Dust-Near and Far. Astromomical Society of the Pacific Conference Series, vol. 414, p. 453 (2009)

Ehrenfreund, P., Charnley, S.B.: Annu. Rev. Astron. Astrophys. 38, $427(2000)$

Elsila, J.E., Glavin, D.P., Dworkin, J.P.: Meteorit. Planet. Sci. 44, 1323 (2009)

Esmaili, S., Bass, A.D., Cloutier, P., Sanche, L., Huels, M.A.: J. Chem. Phys. 147, 224704 (2017)

Fayolle, E.C., Öberg, K.I., Jørgensen, J.K., Altwegg, K., Calcutt, H., Müller, H.S.P., Rubin, M., van der Wiel, M.H.D., Bjerkeli, P., Bourke, T.L., Coutens, A., van Dishoeck, E.F., Drozdovskaya, M.N., Garrod, R.T., Ligterink, N.F.W., Persson, M.V., Wampfler, S.F. (Rosina Team): Nat. Astron. 1, 703 (2017) 
Fitzpatrick, E.L.: Astron. J. 92, 1068 (1986)

Furton, D.G., Witt, A.N.: Astrophys. J. 386, 587 (1992)

Gordon, K.D., Witt, A.N., Friedmann, B.C.: Astrophys. J. 498, 522 (1998)

Gordon, K.D., Clayton, G.C., Misselt, K.A., Landolt, A.U., Wolff, M.J.: Astrophys. J. 594, 279 (2003)

Grebennikova, T.V., Syroeshkin, A.V., Shubralova, E.V., Eliseeva, O.V., Kostina, L.V., Kulikova, N.Y., Latyshev, O.E., Morozova, M.A., Yuzhakov, A.G., Zlatskiy, I.A., Chichaeva, M.A., Tsygankov, O.S.: Sci. World J. 2018, 7360147 (2018)

Harris, M.J., Wickramasinghe, N.C., Lloyd, D., Narlikar, J.V., Rajaratnam, P., Turner, M.P., Al-Mufti, S., Wallis, M.K., Ramadurai, S., Hoyle, F.: Proc. SPIE 4495, 192 (2002)

Herbig, G.H.: Annu. Rev. Astron. Astrophys. 33, 19 (1995)

Hinshaw, G., Larson, D., Komatsu, E., Spergel, D.N., Bennett, C.L., Dunkley, J., Nolta, M.R., Halpern, M., Hill, R.S., Odegard, N., Page, L., Smith, K.M., Weiland, J.L., Gold, B., Jarosik, N., Kogut, A., Limon, M., Meyer, S.S., Tucker, G.S., Wollack, E., Wright, E.L.: Astrophys. J. Suppl. Ser. 208, 19 (2013)

Horneck, G.: Origins of life and evolution of the. Biosphere 23, 37 (1993)

Horneck, G., Bücker, H., Reitz, G.: Adv. Space Res. 14, 41 (1994)

Horneck, G., Eschweiler, U., Reitz, G., Wehner, J., Willimek, R., Strauch, K.: Adv. Space Res. 16, 105 (1995)

Horneck, G., Stöffler, D., Eschweiler, U., Hornemann, U.: Icarus 149, 285 (2001)

Hoyle, F., Wickramasinghe, N.C.: Mon. Not. R. Astron. Soc. 124, 417 (1962)

Hoyle, F., Wickramasinghe, N.C.: Nature 223, 450 (1969)

Hoyle, F., Wickramasinghe, N.C.: Nature 226, 62 (1970)

Hoyle, F., Wickramasinghe, N.C.: Nature 270, 701 (1977)

Hoyle, F., Wickramasinghe, N.C.: Lifecloud: The Origin of Life in the Galaxy. Dent, London (1978)

Hoyle, F., Wickramasinghe, N.C.: Astrophys. Space Sci. 65, 241 (1979)

Hoyle, F., Wickramasinghe, N.C.: In: Ponnamperuma (ed.) Comets and the Origin of Life. Reidel, Dordrecht (1981)

Hoyle, F., Wickramasinghe, N.C.: The Theory of Cosmic Grains. Springer, Heidelberg (1991)

Hoyle, F., Wickramasinghe, N.C.: Astronomical Origins of Life: Steps Towards Panspermia. Kluwer, Dordrecht (2000)

Hoyle, F., Wickramasinghe, N.C., Al-Mufti, S., Olavesen, A.H., Wickramasinghe, D.T.: Astrophys. Space Sci. 83, 405 (1982)

Hughes, D.W.: J. Br. Astron. Assoc. 101, 119 (1991)

Ishii, M., Nagata, T., Sato, S., Watanabe, M., Yao, Y., Jones, T.J.: Astrophys. J. 116, 868 (1998)

Johnson, F.M.: Spectrochim. Acta, Part A, Mol. Biomol. Spectrosc. 65, 1154 (2006)

Junge, K., Eicken, H., Swanson, B.D., Deming, J.W.: Cryobiology 52, 417 (2006)

Knoll, A.H.: Annu. Rev. Earth Planet. Sci. 39, 217 (2011)

Kopparapu, R.K.: Astrophys. J. 767, L8 (2013)

Kuan, Y.-J.: Astrophys. J. 593, 848 (2003)

Kuhn, T.S.: The Structure of Scientific Revolutions. University of Chicago Press, Chicago (1962)

Kwok, S.: Astrophys. Space Sci. 395, 5 (2009)

Kwok, S.: Astron. Astrophys. Rev. 24, 8 (2016)

Kwok, S., Zhang, Y.: Nature 470, 80 (2011)

Laturnus, F.: Environ. Sci. Pollut. Res. 8, 103 (2001)

Laturnus, F., Adams, F.C., Wiencke, C.: Geophys. Res. Lett. 25, 773 (1998)

Li, A., Draine, B.T.: Astrophys. J. 554, 778 (2001)

Lisse, C.M., VanCleve, J., Adams, A.C., A'Hearn, M.F., Fernández, Y.R., Farnham, T.L., Armus, L., Grillmair, C.J., Ingalls, J., Belton, M.J.S., Groussin, O., McFadden, L.A., Meech, K.J., Schultz, P.H., Clark, B.C., Feaga, L.M., Sunshine, J.M.: Science 313, 635 (2006)
Lobert, J.M., Keene, W.C., Logan, J.A., Yevich, R.: J. Geophys. Res. 104, 8373 (1999)

Lynds, B.T., Wickramasinghe, N.C.: Annu. Rev. Astron. Astrophys. 6, 215 (1968)

Matsuura, M., Barlow, M.J., Zijlstra, A.A., Whitelock, P.A., Cioni, M.R.L., Groenewegen, M.A.T., Volk, K., Kemper, F., Kodama, T., Lagadec, E., Meixner, M., Sloan, G.C., Srinivasan, S.: Mon. Not. R. Astron. Soc. 396, 918 (2009)

McFadzean, A.D., Whittet, D.C.B., Bode, M.F., Adamson, A.J., Longmore, A.J.: Mon. Not. R. Astron. Soc. 241, 873 (1989)

Meech, K.J., Weryk, R., Micheli, M., Kleyna, J.T., Hainaut, O.R., Jedicke, R., Wainscoat, R.J., Chambers, K.C., Keane, J.V., Petric, A., Denneau, L., Magnier, E., Berger, T., Huber, M.E., Flewelling, H., Waters, C., Schunova-Lilly, E., Chastel, S.: Nature 552, 378 (2017)

Micelotta, E.R., Jones, A.P., Cami, J., Peeters, E., Bernard-Salas, J., Fanchini, G.: Astrophys. J. 761, 35 (2012)

Muñoz Caro, G.M., Meierhenrich, U.J., Schutte, W.A., Barbier, B., Arcones Segovia, A., Rosenbauer, H., Thiemann, W.H.-P., Brack, A., Greenberg, J.M.: Nature 416, 403 (2002)

Noll, S., Pierini, D., Cimatti, A., Daddi, E., Kurk, J.D., Bolzonella, M., Cassata, P., Halliday, C., Mignoli, M., Pozzetti, L., Renzini, A., Berta, S., Dickinson, M., Franceschini, A., Rodighiero, G., Rosati, P., Zamorani, G.: Astron. Astrophys. 499, 69 (2009)

Okuda, H., Shibai, H., Nakagawa, T., Matsuhara, H., Kobayashi, Y., Kaifu, N., Nagata, T., Gatley, I., Geballe, T.R.: Astrophys. J. 351, 89 (1990)

Omont, A.: Astron. Astrophys. 590, A52 (2016)

Oort, J.H., van de Hulst, H.C.: Bull. Astron. Inst. Neth. 10, 187 (1946)

Prevot, M.L., Lequeux, J., Prevot, L., Maurice, E., Rocca-Volmerange, B.: Astron. Astrophys. 132, 389 (1984)

Rappaport, S., Vanderburg, A., Jacobs, T., LaCourse, D., Jenkins, J., Kraus, A., Rizzuto, A., Latham, D.W., Bieryla, A., Lazarevic, M., Schmitt, A.: Mon. Not. R. Astron. Soc. 474, 1453 (2018)

Sagan, C., Khare, B.N.: Nature 277, 102 (1979)

Scoville, N., Faisst, A., Capak, P., Kakazu, Y., Li, G., Steinhardt, C.: Astrophys. J. 800, 108 (2015)

Seaton, M.J.: Mon. Not. R. Astron. Soc. 187, 73 (1979)

Shivaji, S., Chaturvedi, P., Begum, Z., Pindi, P.K., Manorama, R., Padmanaban, D.A., Shouche, Y.S., Pawar, S., Vaishampayan, P., Dutt, C.B.S., Datta, G.N., Manchanda, R.K., Rao, U.R., Bhargava, P.M., Narlikar, J.V.: Int. J. Syst. Evol. Microbiol. 59, 2977 (2009)

Smith, J.D.T., Draine, B.T., Dale, D.A., Moustakas, J., Kennicutt, R.C. Jr., Helou, G., Armus, L., Roussel, H., Sheth, K., Bendo, G.J., Buckalew, B.A., Calzetti, D., Engelbracht, C.W., Gordon, K.D., Hollenbach, D.J., Li, A., Malhotra, S., Murphy, E.J., Walter, F.: Astrophys. J. 656, 770 (2007)

Sojo, V., Herschy, B., Whicher, A., Camprubí, E., Lane, N.: Astrobiology 16, 181 (2016)

Srinivasan, S., Boyer, M.L., Kemper, F., Meixner, M., Sargent, B.A., Riebel, D.: Mon. Not. R. Astron. Soc. 457, 2814 (2016)

Steele, E.J., Al-Mufti, S., Augustyn, K.A., Chandrajith, R., Coghlan, J.P., Coulson, S.G., Sudipto Ghosh, S., Gillman, M., Gorczynski, R.M., Brig Klyce, B., Louis, G., Mahanama, K., Oliver, K.R., Padron, J., Qu, J., Schuster, J.A., Smith, W.E., Snyder, D.P., Steele, J.A., Stewart, B.J., Temple, R., Tokoro, G., Tout, C.A., Unzicker, A., Wainwright, M., Wallis, J., Wallis, D.H., Wallis, M.K., Wetherall, J., Wickramasinghe, D.T., Wickramasinghe, J.T., Chandra Wickramasinghe, N.C., Liu, Y.: Prog. Biophys. Mol. Biol. 136, 3 (2018)

Strother, P.K., Battison, L., Brasier, M.D., Wellman, C.H.: Nature 473, $505(2011)$

Tamara, D., Lineweaver, C.H.: Publ. Astron. Soc. Aust. 21, 97 (2004)

Thaddeus, P.: Philos. Trans. R. Soc. Lond. B, Biol. Sci. 361, 1681 (2006)

Thiel, C.S., Tauber, S., Schütte, A., Schmitz, B., Nuesse, H., Moeller, R., Ulrich, O.: PLoS ONE 9, e112979 (2014) 
van de Hulst, H.C.: Light Scattering by Small Particles. Wiley, New York (1957)

Vanýsek, V., Wickramasinghe, N.C.: Astrophys. Space Sci. 3, L19 (1975)

Wainwright, M., Wickramasinghe, N., Narlikar, J., Rajaratnam, P.: FEMS Microbiol. Lett. 218, 161 (2003)

Wallis, M.K.: Nature 284, 431 (1980)

Wallis, M.K., Wickramasinghe, N.C.: Mon. Not. R. Astron. Soc. 348, 52 (2004)

Walsh, C., Millar, T.J., Nomura, H., Herbst, E., Widicus Weaver, S., Aikawa, Y., Laas, J.C., Vasyunin, A.I.: Astron. Astrophys. 563, 33 (2014)

Watling, R., Harper, D.B.: Mycol. Res. 102, 769 (1998)

Weissman, P.R.: Astron. Astrophys. 118, 90 (1983)

Wickramasinghe, N.C.: Nature 252, 462 (1974)

Wickramasinghe, N.C.: Search for Our Cosmic Ancestry. World Scientific, London (2015)

Wickramasinghe, D.T., Allen, D.A.: Nature 287, 518 (1980)

Wickramasinghe, D.T., Allen, D.A.: Nature 323, 44 (1986)

Wickramasinghe, N.C., Guillaume, C.: Nature 207, 366 (1965)

Wickramasinghe, N.C., Hoyle, F.: Astrophys. Space Sci. 268, 379 (1999)

Wickramasinghe, N.C., Hoyle, F., Lloyd, D.: Astrophys. Space Sci. 240, 161 (1996)
Wickramasinghe, J.T., Wickramasinghe, N.C., Wallis, M.K.: Int. J. Astrobiol. 8, 281 (2009)

Wickramasinghe, N.C., Wainwright, M., Smith, W.E., Tokoro, G., AlMufti, S., Wallis, M.K.: J. Astrobiol. Outreach 3, 126 (2015)

Wickramasinghe, N.C., Rycroft, M.J., Wickramasinghe, D.T., Steele, E.J., Wallis, D.H., Temple, R., Tokoro, G., Syroeshkin, A.V., Grebennikova, T.V., Tsygankov, O.S.: Adv. Astrophys. 3, 4 (2018)

Witt, A.N., Schild, R.E.: Astrophys. J. 294, 225 (1985)

Witt, A.N., Vijh, U.P.: In: Witt, A.N., Clayton, G.C., Draine, B.T. (eds.) Proceedings of the Conference Held 26-30 May, 2003 in Estes Park, Colorado. Astronomical Society of the Pacific Conference Series, vol. 309, p. 115 (2004)

Witt, A.N., Schild, R.E., Kraiman, J.B.: Astrophys. J. 281, 708 (1984)

Witt, A.N., Mandel, S., Sell, P.H., Dixon, T., Vijh, U.P.: Astrophys. J. 679, 497 (2008)

Wyatt, M.C., van Lieshout, R., Kennedy, G.M., Boyajian, T.S.: Mon. Not. R. Astron. Soc. 473, 5286 (2018)

Yabushita, S.: Mon. Not. R. Astron. Soc. 260, 819 (1993)

Zhang, Y., Kwok, S.: Astrophys. J. 728, 37 (2015)

Zhang, Y., Kwok, S., Sadjadi, S.: J. Phys. Conf. Ser. 728, 052004 (2016)

Zhu, C., Lü, G., Wang, Z.: Mon. Not. R. Astron. Soc. 451, 1561 (2015) 